\title{
Patients with high inflammatory tendency induced by malignant stimulation through imbalance of CD28 and CTLA-4 / PD-1 leading to dopamine neuron injury
}

\section{Li Dong}

Fourth Affiliated Hospital of China Medical University

\section{Yumin Zheng}

The First Affiliated Hospital of China Medical University

Xiaoguang Luo ( $\nabla$ grace_shenyang@163.com )

Shenzhen People's Hospital

Zhiyi He ( $\nabla$ hezy@cmu1h.com )

The First Affiliated Hospital of China Medical University https://orcid.org/0000-0001-7215-1682

\section{Research}

Keywords: high inflammatory tendency, malignant stimulation, CD28, CTLA-4 / PD-1, dopamine neuron injury

Posted Date: February 18th, 2021

DOI: https://doi.org/10.21203/rs.3.rs-208292/v1

License: (9) This work is licensed under a Creative Commons Attribution 4.0 International License. Read Full License 


\section{Abstract \\ Background}

Parkinson's Disease is a common neurodegenerative disease in elderly. Parkinson's Disease patients were supposed to have a higher risk of malignant transformation, however, actually the incidence of various cancers in Parkinson's Disease patients is significantly lower than common people.Parkinson's Disease patients are individuals with a high tendency of inflammation, whose peripheral immune represented in an activated state. The secretion of inflammatory factors in peripheral blood of Parkinson's Disease patients is significantly increased. We hypothesized that the hyperinflammatory predisposition of Parkinson's Disease patients is one of pathogenesis.

\section{Method:}

DBA/1 mice were used to simulate highly inflammatory individuals, and the carcinogen DEN was treated for malignant stimulation.HE staining was used to observe the formation of lung tumors.Apoptosis of neurons was observed by TUNEL staining.Immunohistochemical and Flow cytometrywere used to observed the expression of CD4, CD28, MHCII, CTLA-4 and PD-1. IBA-1 + iNOS was used to label M1 type microglias, and IBA-1 + Arg1 was used to label M2 type microglias by immunofluorescence. The contents of pro-inflammatory cytokines TNF-a, IL-1 $\beta$ and anti-inflammatory cytokines IL-10 and IL-4 in serum and brain tissues of mice in each group were detected by ELISA.

\section{Results}

DBA/1 mice with high inflammatory tendency showed continuous increasing of peripheral inflammation, promoting of intracranial inflammation, decreasing the tumor incidence and increasing the neurodegeneration under induction of malignant change.CD28 and CTLA-4/PD-1 reduced the T-celldominated inflammatory response, reduce intracerebral inflammatory response, protected the neurodegeneration, and increased the incidence of tumor.Combination of CTLA-4 and PD- 1 blocker can over-activate T cells, worsen peripheral and intracranial inflammation, reduce the incidence of tumor, and cause damage to dopamine neurons, promotethe occurrence of neurodegeneration.

\section{Conclusions}

High inflammatory tendency induced by malignant stimulation through imbalance of CD28 and CTLA-4 / PD-1 leading to dopamine neuron injury.

\section{Introduction}


Parkinson's Disease (PD) is a common neurodegenerative disease in elderly. The epidemiological studies of PD demonstrated that the occurrence of PD is contrary to the incidence of malignant tumors; The incidence of lung cancer[1], colon cancer[2] and other cancers in PD patients is significantly lower than that of common people[3], suggesting that individuals with PD tendency perform stronger cancer resistance. On the other hand, cancer is closely related to PD, some pathogenic genes of PD are also oncogenes. Parkin, PINK1 and SNCA are not only pathogenic genes of Parkinson's disease, but also closely related to the incidence of various malignant tumors such as ovarian cancer, lung cancer and liver cancer[4-7]. PD patients were supposed to have a higher risk of malignant transformation, however, actually the incidence of various cancers in PD patients is significantly lower than common people.

PD patients are individuals with a high tendency of inflammation, whose peripheral immune represented in an activated state. The secretion of inflammatory factors in peripheral blood of PD patients is significantly increased[8], the multiple inflammation-related receptors are over-expressed on peripheral immune cells, including antigen-presenting protein MHCIl. These Phenomena suggest the abnormal activation of peripheral immunity in PD patients. Moreover, it has been found that many pathogenic proteinsof PD are actively involved in inflammatory pathways and homeostasis of inflammation[9]. In addition, as the genome sequencing of more than 1,000 PD patients, 24 gene loci were detected to be related with the risk of $\mathrm{PD}$, among which 14 loci were confirmed to be involved in the regulation of immune inflammation[10].

We hypothesized that the hyperinflammatory predisposition of PD patients is one of pathogenesis.People with high inflammatory tendency under stimulation by external malignant change could produce excessive inflammatory response, preventing the occurrence of tumors, however causing the neurodegenerative damage in the brain.

In this study, DBA/1 mice were used to simulate highly inflammatory individuals, and the carcinogen diethylnitrosamine (DEN) was treated for malignant stimulation. DBA/ 1 is an inbred mouse and widely used in immune inflammation studies. DBA/1mice are spontaneously form of arthritis and also used to prepare the collagen induced arthritis model. DBA/1 mice are used to establish the inflammation model of central nervous system, having a high tendency of inflammation background. Compared with C57BL/6, the incidence of tumor in DBA/1 was reduced, however, the damage of dopaminergic neurons in the brain was significantly increased.Moreover, the T-cell-dominated inflammatory response was enhanced by the administration of CTLA-4 and PD-1 inhibitors, leading to a reduced incidence of tumor and enhanced death and neurodegeneration of dopamine neurons. This study demonstrated a close relationship among the high inflammation, tumor, and neurodegeneration, as well as regulating inflammation on the outcome of cancer and neurodegeneration.

\section{Material And Methods}

\section{Laboratory animals and groups}


50 DBA/1 mice and 50 C57BL/6 mice, SPF grade, male, 6-8 weeks, were purchased from Beijing Vital River Laboratory Animal Technology Co., Ltd. (SCXK(Beijing)201200070). Mice were raised in the Laboratory Animal Department of China Medical University (SYXK(Liao) 201880005). The animal experiment was approved by the Ethics Committee of Experimental Animal Welfare of China Medical University (IACUC.No.20180001). Mice were divided into DBA/1 untreated group (DBA), C57BL/6 untreated group (C57), DBA/1 treated with diethylnitrosamine (DEN) group (DD), C57BL/6 treated with diethylnitrosamine group (CD), DBA/1+DEN+anti-CTLA-4 and anti-PD-1 combined treatment group (DDCP group), and C57BL/6 +DEN+anti-CTLA-4 and anti-PD-1 combined treatment group (CDCP group).Mice in DD and CD groups were subcutaneously injected with $1 \%$ DEN $56 \mathrm{mg} / \mathrm{kg}$ once a week for eight weeks.Mice in DDCP and CDCP groups were treated by intraperitoneal injection of CTLA-4 inhibitor, ipilimumab, and anti-PD-1,durvalumab, 10mg/Kg at 7, 8, 9, 12, 15, 18, 21, 24, 27 days after DEN injection.

\section{Hematoxylin-eosin (HE) staining}

HE staining was used to observe the formation of lung tumors.At week 4 and week 8 , Blood samples were taken from the orbital vein and sera were immediately isolated.Thenmice in each group were sacrificed by dislocating their cervical spine. Following that, the lung tissue was collected, fixed by paraformaldehyde. Tissue was dehydrated by ethanol at different concentrations, made transparent with xylene, embedded and block mounted in paraffin.Section were cut and stained with hematoxylin- eosin. An OLYMPUS BX43 was used to capture images.

\section{TUNEL}

Apoptosis of neurons was observed by TUNEL staining.Paraffin-embedded sections of brain tissue were cut into $5 \mu \mathrm{m}$ sections and treated with $50 \mathrm{ml}$ TUNEL reaction solution. The sections were incubated at $37^{\circ} \mathrm{C}$ for $60 \mathrm{~min}$, and then added with $50 \mu \mathrm{L}$ streptomycin avidin-horseradish peroxidase solution incubating for 30min. The nuclei were stained with DAPI; After dehydration, the slices were sealed with an anti-fluorescence quenching agent. The fluorescence was detected under a fluorescence microscope and photographed.

\section{Immunohistochemical}

Paraffin-embedded brain tissue wax blocks were sectioned at $5 \mu \mathrm{m}$. The citric acid antigen repair buffer was placed in the microwave oven for antigen repair, and the sections were placed in $3 \%$ hydrogen peroxide solution to block endogenous peroxidase.3\%BSA was added for blocking at room temperature for 30min.Then anti-CD4, anti-CD28, anti-CTLA-4, anti-PD-1 and anti-MHCll antibodies were separately added to examinethe number of infiltrating $T$ cells in the brain. The tissue sections were covered with HRP-labeled secondary antibody and incubated at room temperature for 50min.DBA kit was used for color development, hematoxylin was used to re-stain thenucleus.After gradient alcohol dehydration and sealing, the slices were observed under microscope.

\section{Immunofluorescence}


Brain sections were subjected to deparaffinized, hydrated, antigen repaired ( $0.01 \mathrm{M}$ citrate buffer, $\mathrm{pH} 6.0)$ and goat serum closed at $37^{\circ} \mathrm{C}$ for $30 \mathrm{~min}$. Serum was poured out, and $\mathrm{TH}$ antibody was added to determine the survival of dopamine neurons. IBA-1+ iNOS labeled M1type microglia cells and IBA-1+Arg1 labeled M2type microglia cells were incubated at $4^{\circ} \mathrm{C}$ overnight. The sections were washed with PBS and added with fluorescence-labeled secondary antibody. The sections were incubated at $37^{\circ} \mathrm{C}$ in dark for $30 \mathrm{~min}$ and then washed with PBS.DAPI staining nuclei were added and incubated at room temperature for $10 \mathrm{~min}$. The slices were rinsed by PBS and sealed with anti-fluorescence quenching. Photograph were taken by fluorescence microscope.

\section{ELISA assay}

The contents of pro-inflammatory cytokines TNF-a, IL-1 $\beta$ and anti-inflammatory cytokines IL-10 and IL-4 in serum and brain tissues of mice in each group were detected by ELISA kit.The operation steps were strictly carried out according to the instructions of the kit.

\section{Flow cytometry}

Mice were narcotized, blood was collected from the abdominal aorta, and EDTA anticoagulant was added. Blood lymphocytes of mice were isolated by blood lymphocyte separation kit, the second layer of milky white lymphocytes was carefully moved to another tube.After centrifugation, the collected lymphocyte cells were re-suspended with PBS, and antibodies of CD4, CD28, CTLA-4, PD-1, and $\mathrm{MHCll}$ were added respectively for flow cytometry detection.

\section{Statistical analysis}

SPSS19.0 statistical software (IBM) was used for statistical analysis. The experimental data were expressed as mean \pm standard deviation. The results of the experiments were analyzed using the $\chi 2$ test for ratio rate comparison and one-way analysis of variance for the other comparisons. $P<0.05$ was consideredtobe statistically significant.

\section{Results}

\section{DBA/1 mice with high inflammatory tendency showed decreasing the tumor incidence andcontinuous increasing of peripheral inflammation}

After carcinogenic induction, the tumor incidence of DBA/1 mice was decreased significantly compared with the C57BL/6. HE staining showed that tumors were observed in mice in theboth of CD and DD group (Fig. 1a); After eight weeks treatment by DEN, the tumor incidence was about $50 \%$ in DD groups, and $100 \%$ in CD group. It suggested that the incidence of tumor was reduced in individual with high inflammatory tendency after malignant transformation.Moreover, after DEN induction, the proinflammatory factors TNF- $\alpha$ and IL-1 $\beta$ in peripheral blood of DBA/1 mice were also increased, the antiinflammatory factors IL-10 and IL-4 were persistently decreased.Interesting, in C57BL/ 6 mice, TNF-a and 
IL-1 $\beta$ were increased firstly and then decreased, IL-10 and IL-4 were decreased firstly and then increased (Fig. 1b).

\section{DBA1 1 mice with high inflammatory tendency showed promoting of intracranial inflammation and increasing the neurodegeneration under induction of malignant change}

After carcinogenic induction, microglia cells in the brain of DBA/1mice were significantly activated.M1 type microglia cells were dominant in DBA/1 mice, M2type microglia cells were relatively reduced. Moreover,M1type microglia cells were significantly increased in DD group compared with the control group. In this study, IBA-1 + iNOS was used to label M1type microglias, and IBA-1 + Arg1 was used to label M2type microglias by immunofluorescence, and the results showed that the activation of microglia in DBA/1 mice was more than that in C57BL/6 mice (Fig. 2a). The damage of dopamine neurons in DBA/1 mice was moresignificant than that in C57BL/ 6 mice under DEN induction.TH staining showed that the number of dopamine neurons in DBA/1 mice was significantly reduced compared with that in C57BL/6 mice (Fig. 2b). Tunel staining assay demonstratedthat the apoptosis of substantia nigra neurons of DBA/1 mice were higher than that of C57BL/6 mice (Fig. 2C).

\section{CD28 and CTLA-4/PD-1 reduced theT-cell-dominated inflammatory response, reduce intracerebral inflammatory response and protectedthe neurodegeneration}

Peripheral T cells were activated and intracranial inflammatory response was significantly increased in the DD group.Theflow cytometry showed that CD4, CD28 and MHCIl in peripheral blood lymphocytes of DD group were significantly increased, while CTLA-4 and PD-1 were significantly decreased (Fig. 3a).Th1mediated TNF- $\alpha$ and IL-1 $\beta$ levels in the DD group wascontinued to increase, however,Th2-mediated IL-10 and IL-4 levels was decreased continually (Fig. 3b).T cells activationin the DBA/ 1 group was significantly increased, and the expressions of CTLA-4 and PD- 1 were gradually decreased.To detect T cell number in mice brain under DEN stimulation, the expression of CD4, CD28, MHCII, CTLA-4 and PD-1 weremeasuredbyimmunohistochemicalstaining (Fig. 3c). The resultsshowedthat the expressions of CD4, $\mathrm{CD} 28$ and $\mathrm{MHCll}$ in the brain wereincreasedgraduallywith time, while the expression of CTLA-4 and PD1 wereedecreasedovertime in DBA/1 micecomparedto C57BL/ 6 mice. These results suggest that activated $T$ cells participate and worsen intracranial inflammation.

\section{Combination of CTLA-4 and PD-1 blocker can over-activate T cells, worsen peripheral and intracranial inflammation, reduce the incidence of tumor}

The combination of CTLA-4 and PD-1 blockers can reduce the incidence of tumors. DBA/1 mice were treated with CTLA-4 and PD-1 blocker after injection of DEN, and the tumor incidenceat 8weekstreatmentwas reduced to $50 \%$ compared with DD group, while the tumor incidence inC57BL/6 mice was about $20 \%$. The combination of CTLA-4 and PD-1 blockers can promote the activation of T cells. The results showedthe level of CD4, CD28, MHCII in peripheral lymphocytes were significantly increased in DDCP group and CDCP groupcompared to thatin the DD and CD group, however, the content of CTLA 4, PD-1 were significantly reduced (Fig. 4a), the changes in the DDCP group were the most 
evident. Furthermore, Immunohistochemistry results demonstrated the similar results that the expression of CD4, CD28 and MHCI in mice brain tissues was increased, while the content of CTLA-4 and PD1 was decreased in theDDCP group and CDCP group compared to that in the DD and CD group(Fig. 4b).Furthermore,ELISA results showed that CTLA-4 and PD-1 blockers could activate the inflammatory response after malignant stimulation in DBA/1, with increased TNF-averify and IL-1 $\beta$ levels and decreased IL-10 and IL-4 levels (Fig. 4c).

\section{Combination of CTLA-4 and PD-1 blocker can cause damage to dopamine neurons, promote the occurrence of neurodegeneration}

Under the combination treatment of CTLA-4 and PD-1 blockers, brain microglia cells were activated and dopaminergic neurons were damaged. Immunofluorescence showed that CTLA-4 and PD-1 blockers activated microglia activity in DBA/1 under DEN stimulation, and M1type microglia was increased, M2type microglia was decreased (Fig. 5a).At the same time, compared with the DD group, the number of dopamine neurons in the DDCP group was decreased (Fig. 5b), and the apoptosis rate of substantia nigra neurons was increased (Fig. 5c).

\section{Discussion}

Parkinson's disease is a common neurodegenerative disease,however,its etiology and pathogenesis are still unclear. PD is considered to be the result of the interaction of multiple factors. In this study, we demonstrated that harmful stimulation induced the individuals with a high tendency of inflammationtoactivate the T cells immune response, increase the stimulating factor, decrease the inhibitory factor, leading to excessive inflammation, spreading to the brain, eventually leading to the damage of neurons. Because the co-inhibitory molecules CTLA-4 and PD- 1 on T cells can down-regulate the immune response, weinhibited the expression of CTLA-4 and PD-1, T cells was activated. Although the occurrence of tumor was reduced, the inflammatory response and the degenerative injury of neurons were aggravated in the brain. The results suggested that the body with high inflammatory tendency is at high risk of PD, and the disorder of immune mechanism may be one of the importantfactors of PD.

In this study, under malignant stimulation the incidence of tumor in DBA/1 mice was lower than that in C57BL/6, additionally, the peripheral pro-inflammatory cytokines TNF-a and IL-1 $\beta$ were continuously increased, the anti-inflammatory cytokines IL-10 and IL-4 were decreased, and the degeneration and damage of neurons in the brain was significant.In C57BL/ 6 mice, TNF- $\alpha$ and IL-1 $\beta$ reached the highest levels at the fourth week after stimulation and then gradually decreased.Weconsidered thatafterfourweeks' stimulation, theinflammatory responsein wildmicewas initiatedto down-regulated. However, in DBA/ 1 mice the inflammatory response was gradually increased, suggesting that inflammation in high-inflammation mice was deregulated, eventually leading to apoptosis and damage of dopamine neurons.Clinically, the high levels of IL-1 $\beta$, TNF-ahavebeenreported in the serum and cerebrospinalfluid of PDpatients[11].This is consistent with our results, thus it is considered that inflammatory factors may also be involved in the occurrence of central inflammation leading to the 
damage of dopamine neurons. We considered that the tendency of high inflammation in the body is a double-edged sword, which not only inhibits the tumorigenesis, but also initiates the neuronal damage in the brain. The pathogenesis of PD is closely related to inflammatory response. Combined withthe significantly decreasing of tumor incidence in PD patients, we speculate that the partially cause of PD patients is based on high inflammation tendency in the body, the body was induced to inflammatory response as facing to the various malignant stimulation in environment, however the inflammation is out of control, leading tothe death of dopaminergic neurons in the brain.

In this study, under DEN induction, following with CTLA-4 and PD-1 inhibitors treatment, CD4, CD28 and $\mathrm{MHCll}$ in peripheral and intracranial of DBA/1 mice were significantly increased, CTLA-4 and PD1 were significantly decreased, and intracranial pro-inflammatory factors were significantly increased.These results suggested that malignant stimulation could over-activate the $T$ cells in highly inflammatory mice and simultaneously participate in and worsen the intracranial inflammatory response, leading to the occurrence of neurodegenerative injury.T cell activation has always been the research spot of anti-tumor immunity and tumor vaccine research. And T cell activation is also closely related to PD.SaundersEt al. demonstrated that the imbalance between memory $T$ cells and regulatory $T$ cells was closely related to the severity of $\mathrm{PD}[12]$. T cell over-activation is also involved in exacerbating inflammation in the brain.In the studies of PD patient brain[13]and PD animal models[14], a large number of $C^{4+}$ and $C D^{8+} T$ cells were infiltrated. In addition, various treatment in activating T cell immunity to inhibit tumor growth[15] also cause immune encephalitis[16], Transverse myelitis[17]. These studies suggesting that the overactivated $T$ cell immune system in peripheral blood can cause inflammation of the central nervous system.Therefore, we considered that highly inflammatory individuals activate T-cell immunity in order to combat malignant stimuli, triggering the strong peripheral inflammation, inhibiting tumor,however, promoting inflammatory response and neurodegeneration in the brain.

In this study, under DEN induction, following with CTLA-4 and PD-1 inhibitor treatment,the tumor incidence rate in DBA/1 mice was decreased, CD28 content in peripheral blood and brain was increased, and the content of CTLA-4 and PD1 was further decreased. The content of pro-inflammatory factors in peripheral blood and brain tissue was also increased, and the content of anti-inflammatory factors was decreased, therefore the damage and apoptosis of dopamine neurons in brain were more significant.It was suggested that the combined application of CTLA-4 and PD- 1 inhibitor further promoted T cells activation, aggravated the damage of dopamine neurons, and significantly decreased the incidence of tumor.These results showed that CTLA-4 and PD-1 could regulate the process of T cell activation and resist tumorincidence and aggravate the dopamine neuron injury. The activation of $T$ cells requires dual signals, the first signal comes from the specific binding of MHC-antigen complex of Antigen-presenting cells (APC) withT cell receptor (TCR), and the second signal comes from the binding of co-stimulating molecules (CD28) or co-inhibiting molecules (CTLA-4 and PD-1) on the surface of T cells with the ligands on the APC. CD28, a co-stimulatory molecule on the surface of T cells, can activate $T$ cells and promote inflammation after stimulation.The co-inhibitory molecules CTLA-4 and PD-1 can inhibit the activation of inflammatory T cells. CD28, CTLA-4 and PD-1 can precisely regulate the transition of T cells to pro- 
inflammatory Th1 oranti-inflammatory Th2, and affecting the activation state of peripheral immunity. The inhibitor of CTLA-4 performed good treatment efficacyin rheumatoid arthritis[18], Type 1

diabetes[19],Kidney transplantation immune rejection[20].On the contrary, co-inhibitory molecules such as CTLA-4 and PD-1 are increasingly expressed in tumors, which inhibit the activation of T cells and weaken the immune surveillanceof tumors, becoming the most important mechanism for tumor-cell escape[21].This study shows that the combination of CTLA-4 and PD-1 inhibitors can activate T cells in highly inflammation-prone animals and promote excessive inflammation, and increase the transformation of microglia into M1type cells to aggravate the damage of dopamine neurons, promoting the development of PD, however, reducing tumor escape and significantly decreasing the incidence of tumor.

The above results demonstrated that individualswith high inflammatory tendency are the basis for the occurrence of Parkinson's disease. As exposed to harmful stimuli, inflammation is initiated to protect the body from the occurrence of malignant tumors. However, due to their high inflammatory tendency,excessive inflammation is caused and eventually dopamine neurons are injured.The harmful stimulus may be not only confined to DEN, it may also include a variety of microbial infection, chemicals, all kinds of physical and chemical stimulation, etc. These harmful stimuluses induced the excessive inflammation in the individual with high tendency, accumulated gradually in a long period leading to the damage of the central nervous system. This study confirmed that bodywith a high inflammatory tendency are at high risk of excessive inflammatory response to malignant stimulation. In the process, CTLA-4 and PD-1 as important regulatory factors could reduce the inflammatory response.As the block of CTLA-4 and PD-1 together lead to deterioration of inflammation, increasing the damage of neurons in the brain.

Although it could curb the occurrence of tumors, increase the risk of nerve degeneration. These finding confirmed thatthe inflammation tendency of genetic background is the pathogenesis of PD risk factors.

\section{Conclusions}

High inflammatory tendency induced by malignant stimulation through imbalance of CD28 and CTLA-4 / PD-1 leading to dopamine neuron injury.

\section{Abbreviations}

PD Parkinson's Disease

DEN Diethylnitrosamine

HE Hematoxylin-eosin

TUNEL TdT-mediated dUTP Nick-End Labeling

ELISA Enzyme linked immunosorbent assay 
TNF-a Tumor necrosis factor-a

IL-1 $\beta$ Interleukin-1 $\beta$

IL-10 Interleukin-10

TGF- $\beta$ transforming growth factor- $\beta$

IL-4 Interleukin-4

CTLA-4 Cytotoxic T lymphocyte-associated antigen-4

PD-1 Programmed cell death protein 1

EDTA Ethylene Diamine Tetraactic Acid

MHCII Major histocompatibility complex II

\section{Declarations}

\section{Acknowledgements}

Not applicable.

\section{Funding}

This research was supported by the National Nature Science Foundation ofChina (grant no. 81771375).

\section{Availability of data and materials}

Data supporting the conclusions of this article are presented in themanuscript.

\section{Authors' contributions}

ZY-H and LD conceived and designed the experiments. LD, YM-Z, XG-L, andZY-H performed the experiments. LD, YM-Z, XG-L, andZY-H analyzed the data,and LD,XG-L,and ZY-H wrote the paper. All authors read and approved the finalmanuscript.

\section{Ethics approval}

All animal procedures performed in this study were reviewed and approvedbythe animal experiment was approved by the Ethics Committee of Experimental Animal Welfare of China Medical University (IACUC. No. 20180001).

\section{Consent for publication}

Not applicable. 


\section{Competing interests}

The authors declare that they have no competing interests.

\section{References}

1. Xie X, Luo X, Xie M, Liu Y, Wu T. Risk of lung cancer in Parkinson's disease.Oncotarget 2016, 7:77319-77325.

2. Xie X, Luo X, Xie M. Association between Parkinson's disease and risk of colorectal cancer. Parkinsonism Relat Disord. 2017;35:42-7.

3. Tacik P, Curry S, Fujioka S, Strongosky A, Uitti RJ, van Gerpen JA, DiehI NN, Heckman MG, Wszolek ZK. Cancer in Parkinson's disease. Parkinsonism Relat Disord. 2016;31:28-33.

4. Mehdi SJ, Ali A, Rizvi MM. Parkin gene alterations in ovarian carcinoma from northern Indian population. Pathol Oncol Res. 2011;17:579-86.

5. Picchio MC, Martin ES, Cesari R, Calin GA, Yendamuri S, Kuroki T, Pentimalli F, Sarti M, Yoder K, Kaiser LR, et al. Alterations of the tumor suppressor gene Parkin in non-small cell lung cancer. Clin Cancer Res. 2004;10:2720-4.

6. Fujiwara M, Marusawa H, Wang HQ, Iwai A, Ikeuchi K, Imai Y, Kataoka A, Nukina N, Takahashi R, Chiba T. Parkin as a tumor suppressor gene for hepatocellular carcinoma. Oncogene 2008, 27:60026011.

7. Morris LG, Veeriah S, Chan TA. Genetic determinants at the interface of cancer and neurodegenerative disease. Oncogene 2010, 29:3453-3464.

8. Wang XM, Zhang YG, Li AL, Long ZH, Wang D, Li XX, Xia JH, Luo SY, Shan YH. Relationship between levels of inflammatory cytokines in the peripheral blood and the severity of depression and anxiety in patients with Parkinson's disease. Eur Rev Med Pharmacol Sci. 2016;20:3853-6.

9. Dzamko N, Geczy CL, Halliday GM. Inflammation is genetically implicated in Parkinson's disease.Neuroscience 2015, 302:89-102.

10. Nalls MA, Saad M, Noyce AJ, Keller MF, Schrag A, Bestwick JP, Traynor BJ, Gibbs JR, Hernandez DG, Cookson MR, et al. Genetic comorbidities in Parkinson's disease. Hum Mol Genet. 2014;23:831-41.

11. Dobbs RJ, Charlett A, Purkiss AG, Dobbs SM, Weller C, Peterson DW. Association of circulating TNFalpha and IL-6 with ageing and parkinsonism.Acta Neurol Scand 1999, 100:34-41.

12. Saunders JA, Estes KA, Kosloski LM, Allen HE, Dempsey KM, Torres-Russotto DR, Meza JL, Santamaria PM, Bertoni JM, Murman DL, et al. CD4 + regulatory and effector/memory T cell subsets profile motor dysfunction in Parkinson's disease. J Neuroimmune Pharmacol. 2012;7:927-38.

13. Brochard V, Combadiere B, Prigent A, Laouar Y, Perrin A, Beray-Berthat V, Bonduelle O, Alvarez-Fischer $D$, Callebert J, Launay JM, et al. Infiltration of CD4 + lymphocytes into the brain contributes to neurodegeneration in a mouse model of Parkinson disease. J Clin Invest. 2009;119:182-92. 
14. Benner EJ, Banerjee R, Reynolds AD, Sherman S, Pisarev VM, Tsiperson V, Nemachek C, Ciborowski P, Przedborski S, Mosley RL, Gendelman HE. Nitrated alpha-synuclein immunity accelerates degeneration of nigral dopaminergic neurons.PLoS One 2008, 3:e1376.

15. Galaine J, Borg C, Godet Y, Adotevi O. Interest of Tumor-Specific CD4 T Helper 1 Cells for Therapeutic Anticancer Vaccine. Vaccines (Basel) 2015, 3:490-502.

16. Williams TJ, Benavides DR, Patrice KA, Dalmau JO, de Avila AL, Le DT, Lipson EJ, Probasco JC, Mowry EM. Association of Autoimmune Encephalitis With Combined Immune Checkpoint Inhibitor Treatment for Metastatic Cancer.JAMA Neurol 2016, 73:928-933.

17. Liao B, Shroff S, Kamiya-Matsuoka C, Tummala S. Atypical neurological complications of ipilimumab therapy in patients with metastatic melanoma.Neuro Oncol 2014, 16:589-593.

18. Keystone E, Alkhalaf A, Makkawy M. Subcutaneous abatacept in rheumatoid arthritis: current update. Expert Opin Biol Ther. 2015;15:1221-30.

19. Orban T, Bundy B, Becker DJ, Dimeglio LA, Gitelman SE, Goland R, Gottlieb PA, Greenbaum CJ, Marks $\mathrm{JB}$, Monzavi $\mathrm{R}$, et al: Costimulation modulation with abatacept in patients with recent-onset type 1 diabetes: follow-up 1 year after cessation of treatment.Diabetes Care 2014, 37:1069-1075.

20. Del Bello A, Marion O, Milongo D, Rostaing L, Kamar N. Belatacept prophylaxis against organ rejection in adult kidney-transplant recipients. Expert Rev Clin Pharmacol. 2016;9:215-27.

21. Baksh K, Weber J. Immune checkpoint protein inhibition for cancer: preclinical justification for CTLA4 and PD-1 blockade and new combinations.Semin Oncol 2015, 42:363-377.

\section{Figures}

(a)
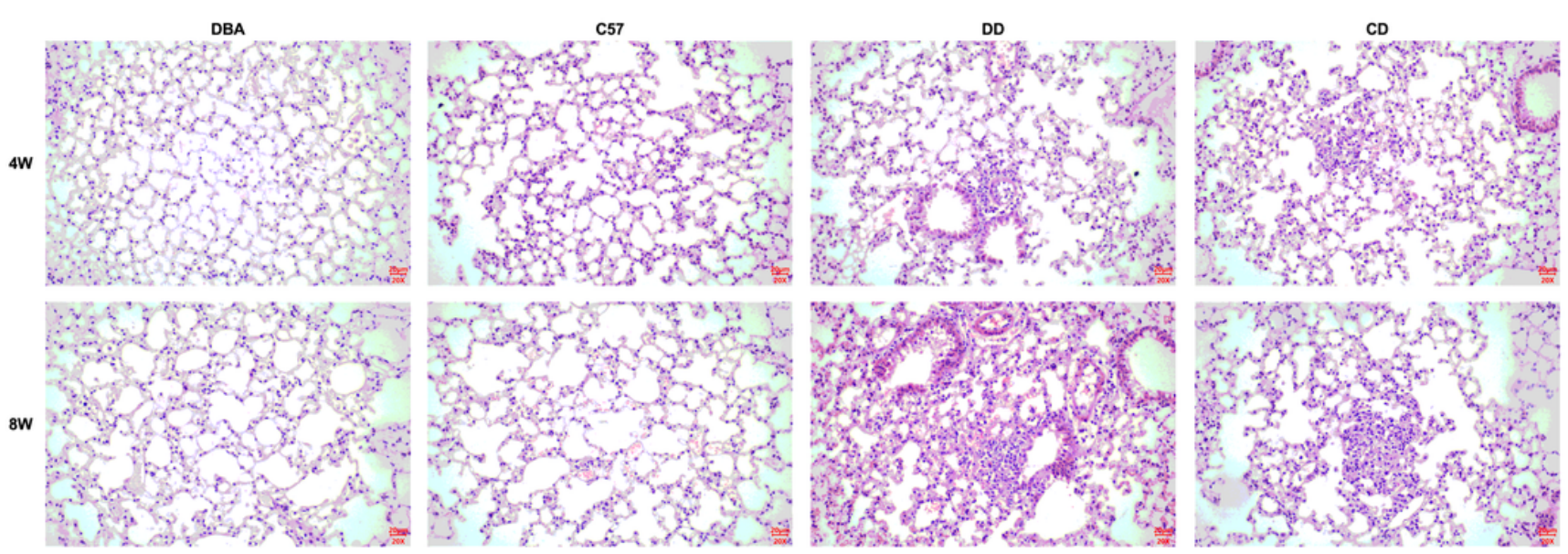

(b)
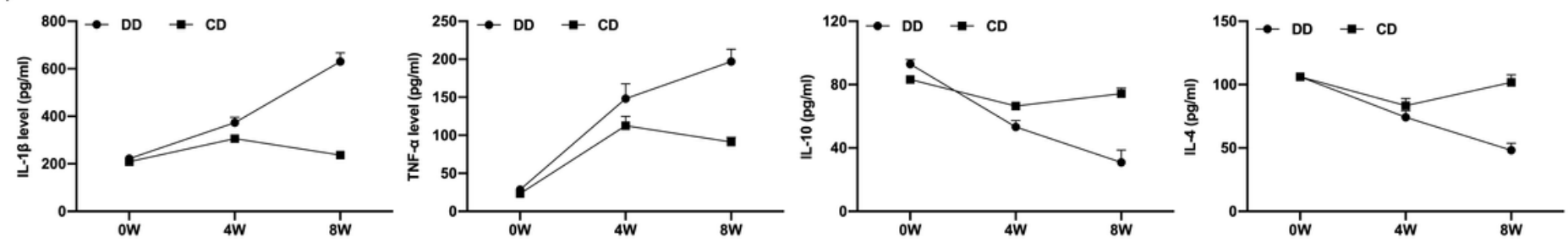
Figure 1

Comparison of the tumor incidence and peripheral inflammation of mice in different groups. The results displayed thattumors were observed in mice in the both of CD and DD group (a). The pro-inflammatory factors TNF- $\alpha$ and IL- $1 \beta$ in peripheral blood of DBA/ 1 mice were also increased, the anti-inflammatory factors IL-10 and IL- 4 were persistently decreased, in C57BL/ 6 mice, TNF- $\alpha$ and IL-1 $\beta$ were increased firstly and then decreased, IL-10 and IL-4 were decreased firstly and then increased (b).

(a)
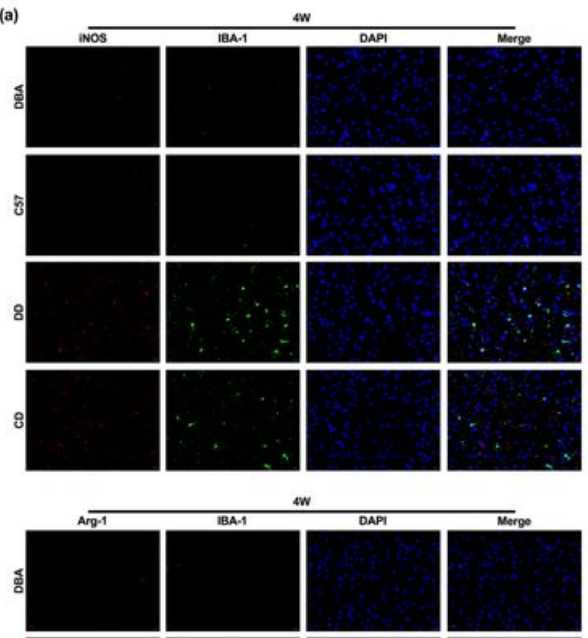

DAPI
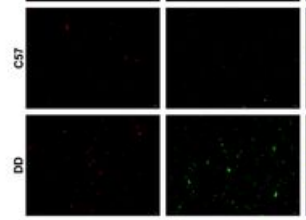

$+2$

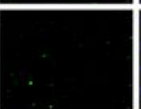

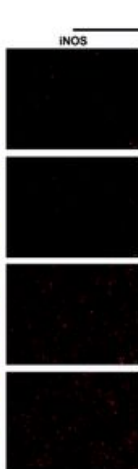
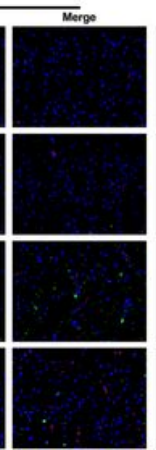

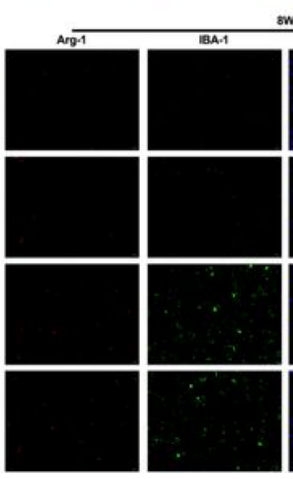

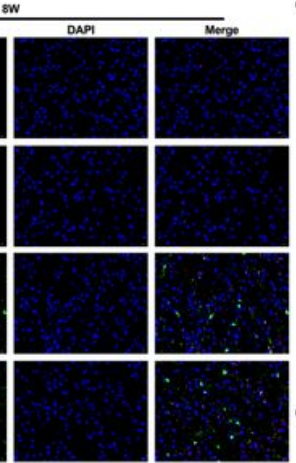
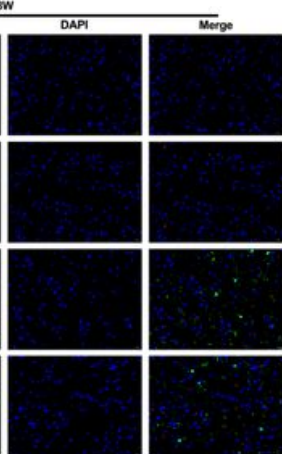
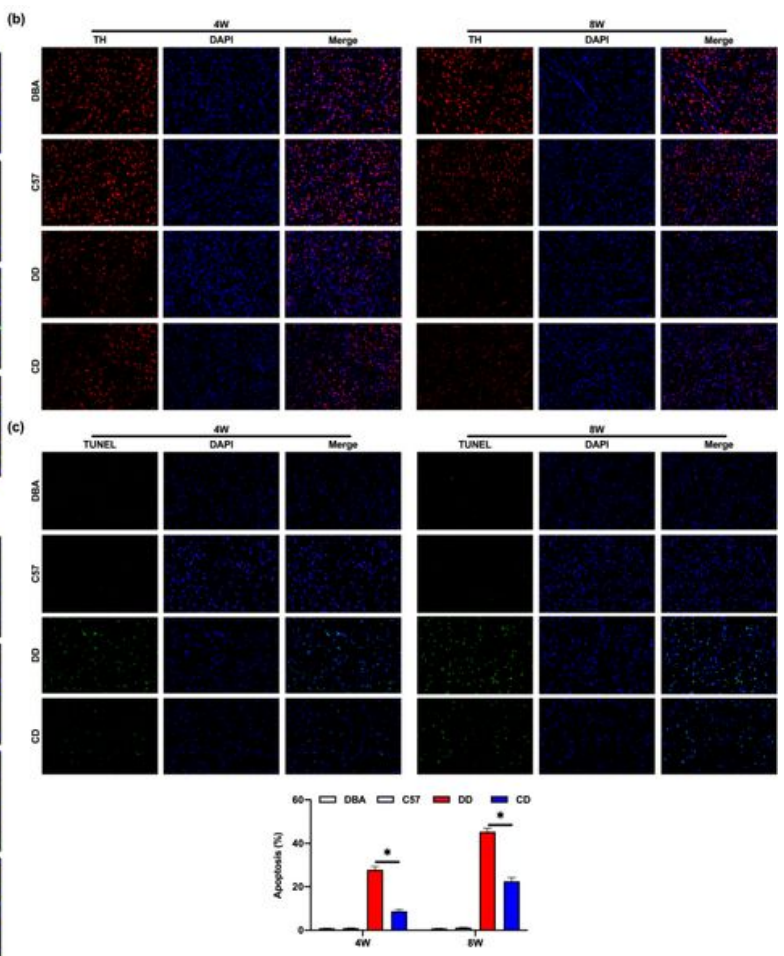

Figure 2

Comparison ofthe intracranial inflammation and neurodegeneration of mice in different groups. The results displayed thatM1 type microglia cells were dominant in DBA/1 mice, M2 type microglia cells were relatively reduced, $\mathrm{M} 1$ type microglia cells were significantly increased in DD group compared with the control group, and the activation of microglia in DBA/1 mice was more than that in C57BL/6 mice (a). TH staining showed that the number of dopamine neurons in DBA/1 mice was significantly reduced compared with that in C57BL/6 mice (b). Tunel staining assay demonstrated that the apoptosis of substantia nigra neurons of DBA/1 mice was higher than that of C57BL/6 mice (c). *indicatedP<0.05. 
(a)
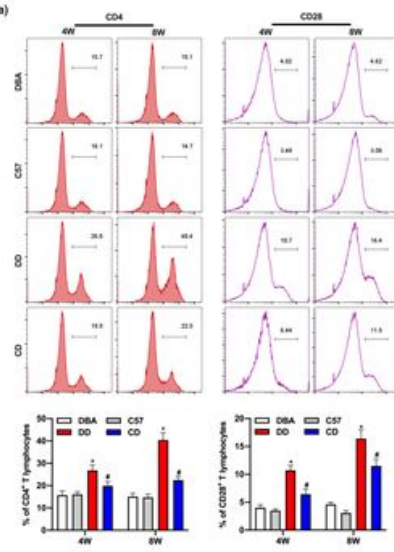
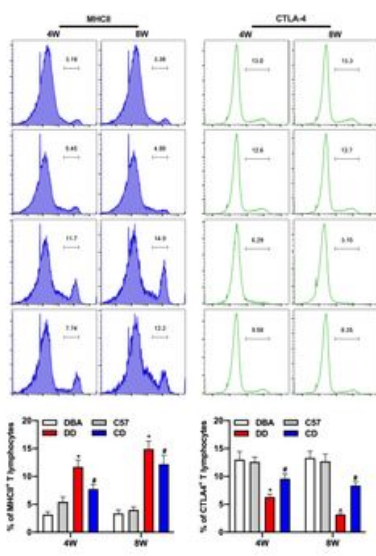
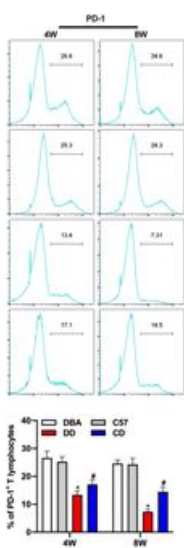
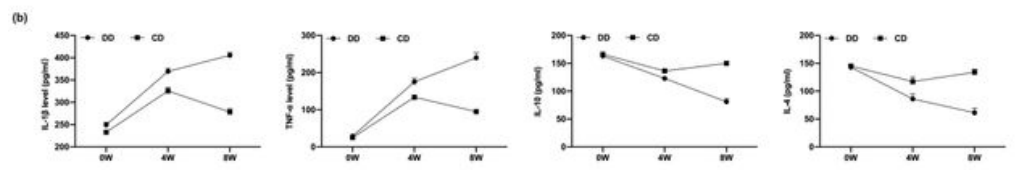
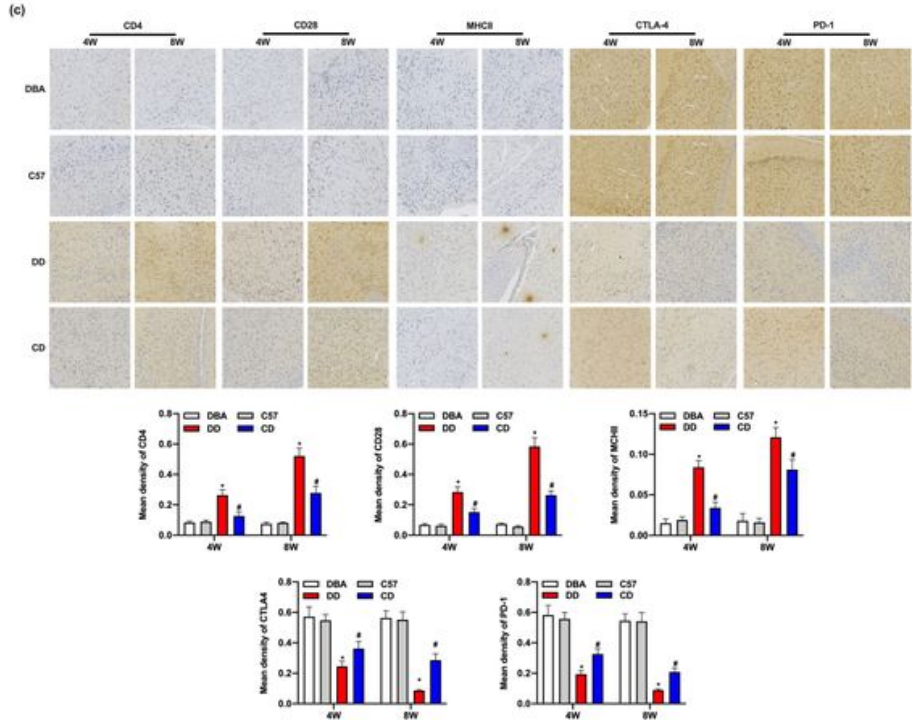

Figure 3

Comparison ofthe intracerebral inflammatory response and neurodegeneration of mice in different groups. The flow cytometry showed that CD4, CD28 and MHCIl in peripheral blood lymphocytes of DD group were significantly increased, while CTLA-4 and PD-1 were significantly decreased (a). Th1-mediated TNF- $\alpha$ and IL-1 $\beta$ levels in the DD group was continued to increase, however, Th2-mediated IL-10 and IL-4 levels was decreased continually (b). Immunohistochemical staining showed that the expressions of $\mathrm{CD} 4, \mathrm{CD} 28$ and $\mathrm{MHCl}$ in the brain were increased gradually with time, while the expression of CTLA-4 and PD-1 were edecreased over time in DBA/1 mice compared to C57BL/6 mice (c).*indicated compare with DBA group, $\mathrm{P}<0.05$; \# indicated compare with $\mathrm{C} 57$ group, $\mathrm{P}<0.05$. 
(a)
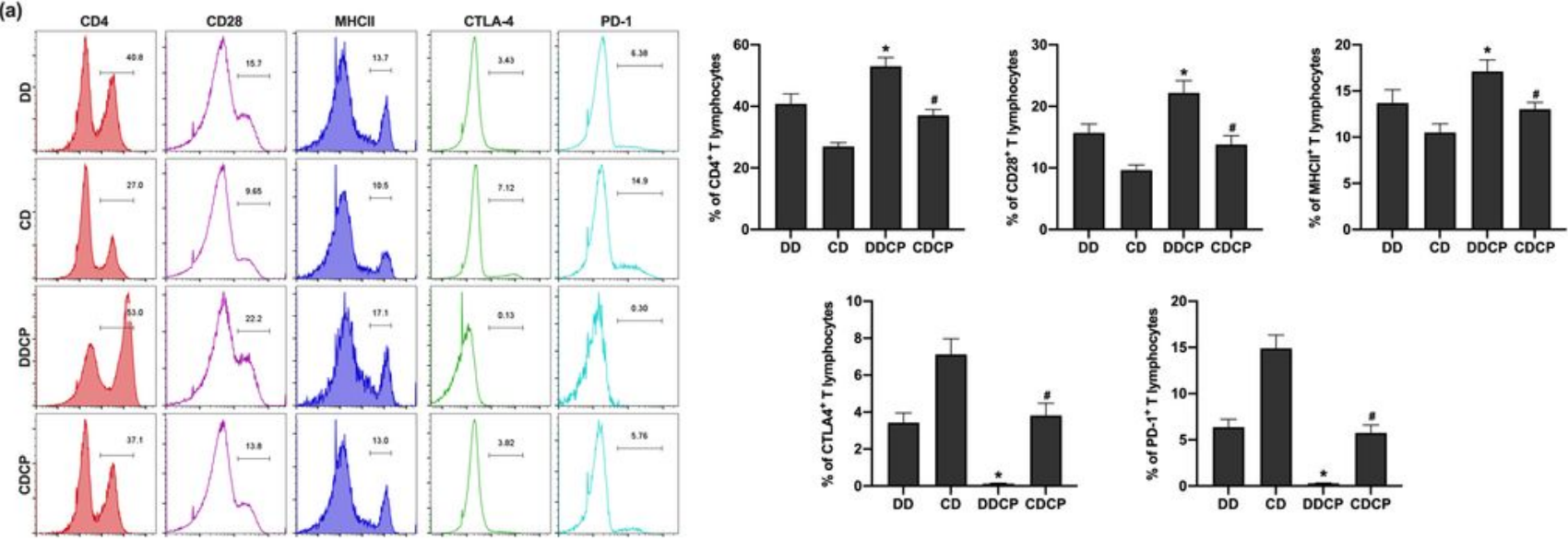

(b)
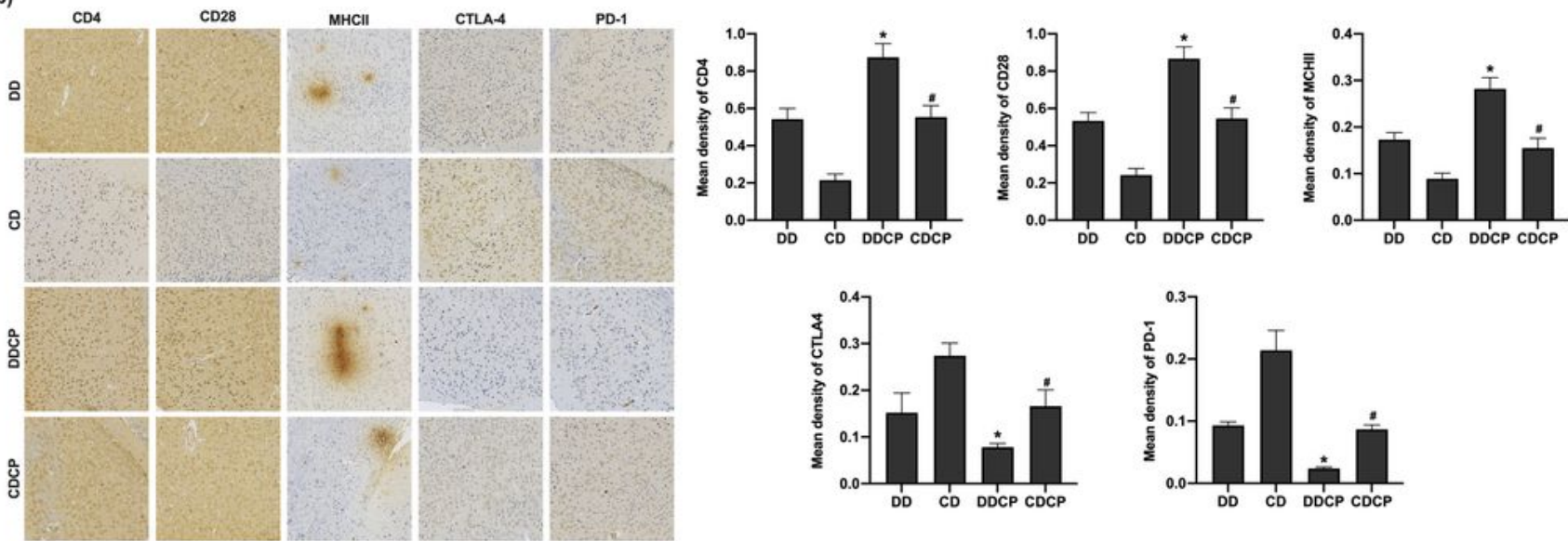

(c)
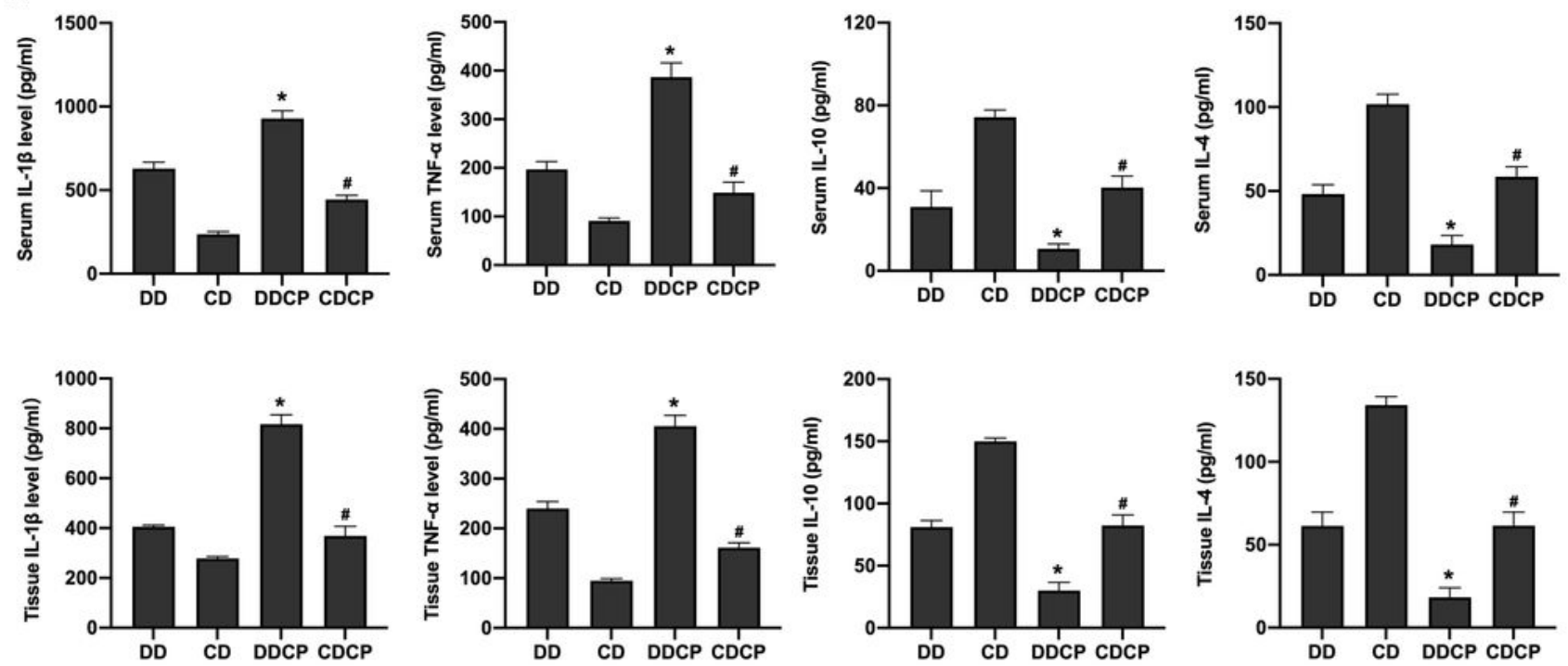

Figure 4

Combination of T cells activate, peripheral and intracranial inflammation and the incidence of tumor amongCTLA-4 and PD-1 blocker treated.The level of CD4, CD28, MHCIl in peripheral lymphocytes were significantly increased in DDCP group and CDCP group compared to that in the DD and CD group, however, the content of CTLA 4, PD-1 were significantly reduced (a). Immunohistochemistry results demonstrated the similar results that the expression of CD4, CD28 and MHCII in mice brain tissues was 
increased, while the content of CTLA-4 and PD1 was decreased in the DDCP group and CDCP group compared to that in the DD and CD group (b). ELISA results showed that CTLA-4 and PD-1 blockers could activate the inflammatory response after malignant stimulation in DBA/1, with increased TNF-averify and IL-1 $\beta$ levels and decreased IL-10 and IL-4 levels (c).*indicated compare with DD group, $P<0.05$; \# indicated compare with CD group, $\mathrm{P}<0.05$.

(a)
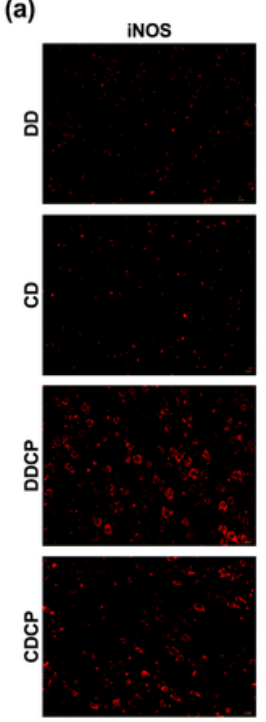

(b)

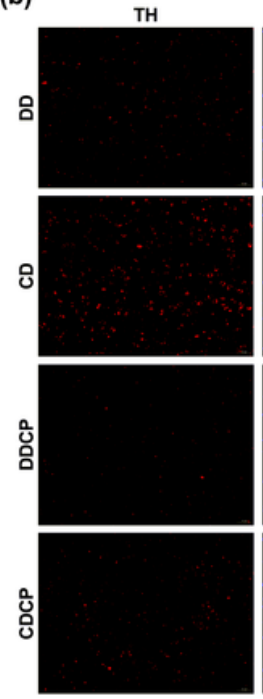

IBA-1
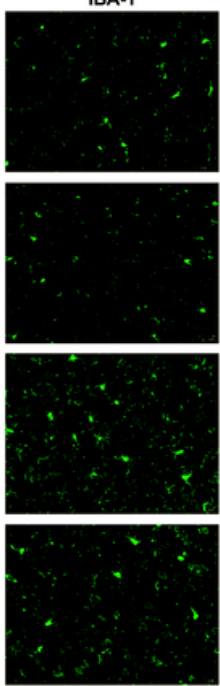

DAPI
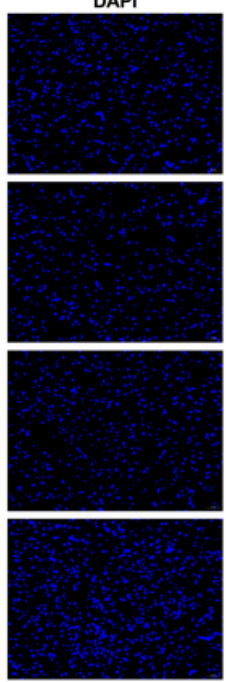

DAPI
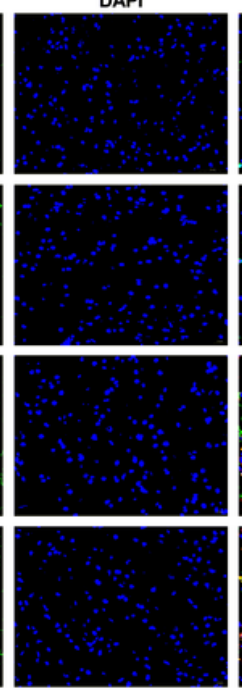

Merge
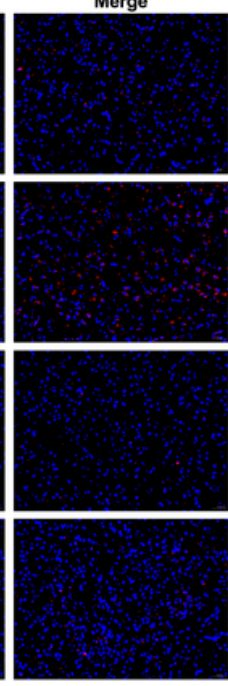
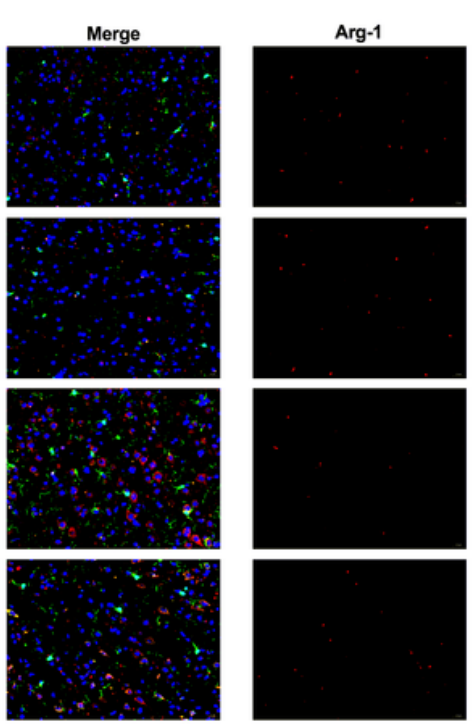

(c)

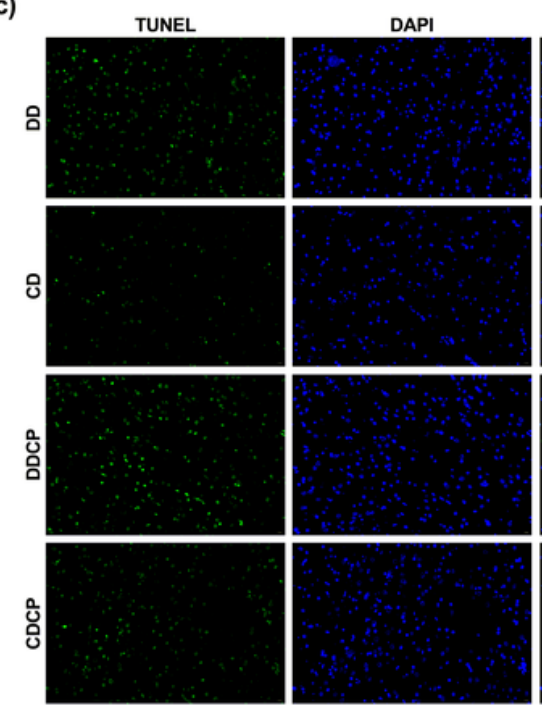

IBA-1
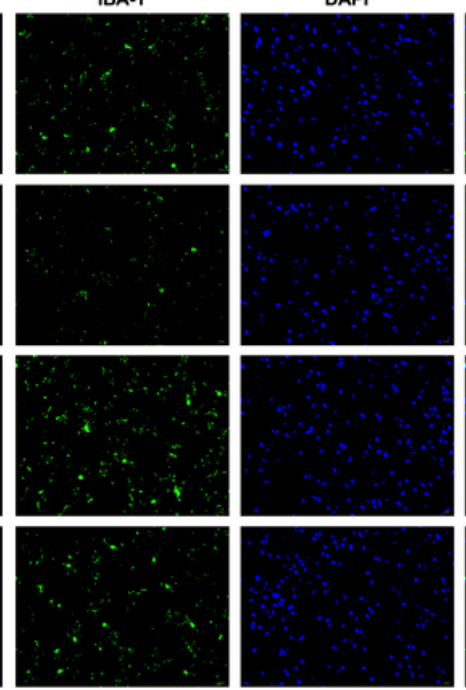

Merge
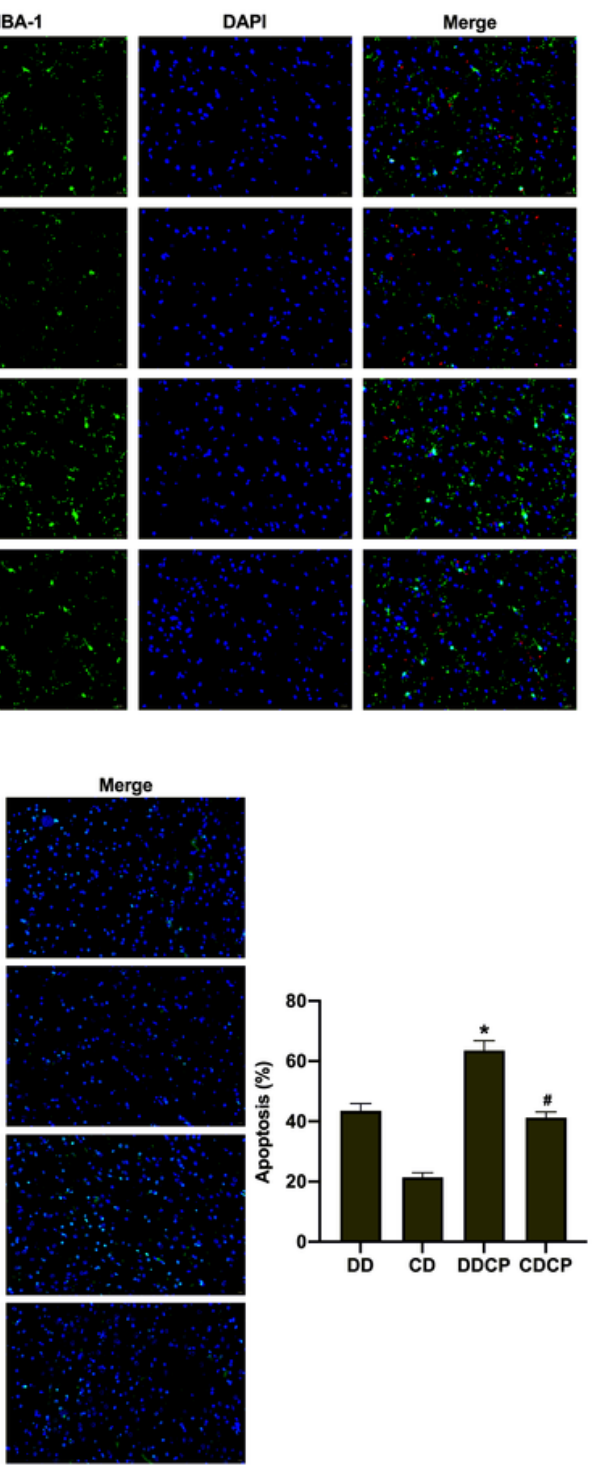

\section{Figure 5}

Combination of damage to dopamine neurons, the occurrence of neurodegeneration among CTLA-4 and PD-1 blocker treated.Immunofluorescence showed that CTLA-4 and PD-1 blockers activated microglia activity in DBA/1 under DEN stimulation, and M1 type microglia was increased, M2 type microglia was decreased (a). TH staining showed that compared with the DD group, the number of dopamine neurons in the DDCP group was decreased (b). Tunel staining assay demonstrated thatcompared with the DD group,the apoptosis rate of substantia nigra neurons was increased in the DDCP group(c).*indicated compare with DD group, $\mathrm{P}<0.05$; \# indicated compare with $C D$ group, $P<0.05$. 